ARTICLE

DOI: $10.1038 / s 41467-017-02034-w$

\title{
Tree co-occurrence and transcriptomic response to drought
}

\author{
Nathan G. Swenson', Yoshiko lida², Robert Howe ${ }^{3}$, Amy Wolf ${ }^{3}$, María Natalia Umaña (1) ${ }^{1}$, Krittika Petprakob ${ }^{1}$,
} Benjamin L. Turner (D) ${ }^{4}$ \& Keping $M a^{5}$

The distribution and co-occurrence of species are partly the outcome of their interactions with environmental drivers. Drought is a key driver related to the distribution of plant species. Drought events continue to increase in frequency and severity and identifying those aspects of plant function that are related to drought is critical. Here, we perform a community-level analysis of gene expression in relation to experimental drought and relate the similarity in gene set enrichment across species to their natural co-occurrence. Species with similar gene set enrichment in response to experimental drought tend to non-randomly co-occur in a natural stand. We demonstrate that similarity in the transcriptomic response of species to drought is a significantly better indicator of natural co-occurrence than measures of functional trait similarity and phylogenetic relatedness and that transcriptomics has the capacity to greatly enhance ecological investigations of species distributions and community structure.

\footnotetext{
${ }^{1}$ Department of Biology, University of Maryland, College Park, MD 20742, USA. ${ }^{2}$ Kyushu Research Center, Forestry and Forest Products Research Institute, Kumamoto, 860-0862, Japan. ${ }^{3}$ Department of Natural and Applied Sciences, University of Wisconsin-Green Bay, Green Bay, WI 54311, USA. ${ }^{4}$ Smithsonian Tropical Research Institute, Apartado, 0843-03092 Balboa, Ancon, Republic of Panama. ${ }^{5}$ State Key Laboratory of Vegetation and Environmental Change, Institute of Botany, The Chinese Academy of Sciences, Beijing, 100093, China
} 
F nvironmental drivers interface with organismal function to determine individual demographic performance that scales up to partly determine species distributions through space and time ${ }^{1}$. Drought is known to be a key driver of tree species distributions and forest functioning from local to regional spatial scales ${ }^{2,3}$. Drought events have massive impacts on forest demography and ultimately carbon flux ${ }^{4,5}$. The strength and periodicity of drought events are expected to increase as regional climates undergo change ${ }^{6}$. In order to predict the effects of droughtinduced change in forests, tree ecologists must first identify those aspects of plant function that best indicate drought response and to link those aspects with the distributions and demography of species in natural stands.

The way in which species respond to drought should influence their spatial co-occurrence in one of two ways. First, species with similar responses should tend to co-occur. This may be caused when less drought tolerant species exhibit superior performance in wetter environments, thereby excluding more drought tolerant species that will dominate drier environments. This mechanism may be manifested at both local and regional scales ${ }^{2}$. Alternatively, species with dissimilar strategies in response to drought (i.e., tolerators vs. avoiders) may be expected to co-occur at a higher frequency due to a reduction in direct competition during periods of low water resource availability. This mechanism may be more likely to occur in areas with strong seasonality in precipitation (i.e., deserts and tropical dry forests), compared with a seasonal rain forests or temperate deciduous forests.

There has been a marked increase in the number of studies that seek to elucidate the environmental drivers of species distributions and community structure ${ }^{1,7-9}$ by quantifying functional traits believed to be representative of the major axes of plant ecological strategy ${ }^{10,11}$. In concept, functional traits are traits directly related to the molecular functions or biological processes that impact performance or fitness ${ }^{1}$. However, in practice, typically only a few functional traits are measured and these traits are often only loosely or indirectly related to the physiological processes or rates of interest. In addition, these traits are representative of only a small portion of the diversity of functions in a plant $^{9}$. A further limitation of functional trait-based ecology is that it generally assigns static trait values to individuals or species, thereby failing to capture the dynamic responses of an organism to important and temporally dynamic environmental drivers such as water availability.

In recognition of the limitations to the functional trait-based approach where important unmeasured traits and/or complex phenotypes such as response to drought are not well-estimated, some have proposed that phylogenetic information can be utilized as a way to estimate the unmeasured ecological similarity between species $^{12,13}$. This approach, however, critically relies on an unknown degree of phylogenetic signal in function, and even in the best-case scenario it is a blind approach where the functional mechanisms driving the phylogenetic pattern will never be known. Combined, the above limitations may explain in part why functional trait and phylogenetically-based studies of plant distributions and community structure often uncover patterns that are weak or do not clearly point to a mechanism ${ }^{7,14-16}$. It is therefore likely that functional trait-based and phylogenetic investigations omit a great deal of mechanistic detail. Specifically, detail regarding how key environmental drivers such as drought and organismal function interface to determine the spatial distribution and co-occurrence of species.

Here, via a community-level analysis of gene expression in relation to experimental drought, we demonstrate that similarity in the transcriptomic response of species to drought is a significantly better indicator of natural co-occurrence than measures of functional trait similarity and phylogenetic relatedness.

\section{Results}

Transcriptomic response of seedlings to experimental drought. Here we quantified the dynamic physiological response of tree seedlings from 21 species to experimental drought through the investigation of transcriptomic data and asked whether gene expression to experimental drought was related to the cooccurrence of these species in a topographically heterogeneous long-term forest dynamics plot in northern Wisconsin, USA (Supplementary Table 1). The forest plot is $25.2 \mathrm{ha}$ in area and we analyzed the $63020 \times 20 \mathrm{~m}$ subplots in the plot where all freestanding woody stems $1 \mathrm{~cm}$ or greater in diameter are tagged, mapped, measured, and identified. The plot has a wellcharacterized soil moisture gradient (Methods) with low elevations in the plot that have standing water during the late spring and early summer and higher elevations with more xeric soils. To test the perceived importance of this gradient and to integrate transcriptomic information into community ecology, we exposed 1-year old seedlings from these species collected from the region to experimental drought in the greenhouse and quantified differential gene expression between control and droughted plants for each species (Methods). We acknowledge that our forest plot data are from individuals far larger than seedlings and that this is a limitation of the study. However, we also note that previous work has demonstrated that differential demography at the seedling stage in response to abiotic and biotic conditions leaves a lasting imprint on juvenile and adult tree assemblages ${ }^{2}$. Next, for each of these species we quantified gene set enrichment, ignoring the directionality of the change in expression, for shared molecular function and biological process gene ontology (GO) terms identified across species. This approach enabled us to compare similar functions and processes across species without having to identify shared individual homologous genes across species. It is important to note that only GO categories identified across all species were used in this study to facilitate an unbiased comparison. A downside of this approach is that it relies on annotations derived from potentially distantly related model species, which could limit the scope of valid inferences ${ }^{17}$. Next, we then clustered species by their similarity in gene set enrichment across all molecular functions or biological processes (Supplementary Figs. 1,2). This allowed us to generate a metric of overall similarity in expression similarity to drought across all species via branch lengths on the hierarchical clustering dendrogram. In sum, we were able to quantify the inter-specific similarity in gene set enrichment in response to experimental drought for $\mathrm{GO}$ biological processes and GO molecular functions.

Transcriptomic response correlates with tree co-occurrence. Combined with the observed natural spatial distributions of the 21 species and null modeling of species co-occurrence patterns, the transcriptomic information enabled us to quantify whether overall similarity in gene set enrichment in response to drought was non-randomly associated with natural tree species cooccurrence and to identify which individual molecular functions and biological processes were most strongly linked to natural tree species co-occurrence.

Next, we contrasted our gene expression analyses with analyses of the number of days to wilting in our drought experiment (Methods) as well as analyses of the functional trait and phylogenetic relatedness of the 21 species. In our previous work $^{18}$, we have quantified eight commonly measured functional traits (leaf $\% \mathrm{C}, \% \mathrm{~N}, \% \mathrm{P}$, wood density, leaf area, specific leaf area, maximum height, and seed mass) and have constructed a molecular phylogeny containing these 21 species $^{19}$. Using the wilting, trait, and phylogenetic data, the spatial distributions of the 21 species in the forest and null models, we quantified 


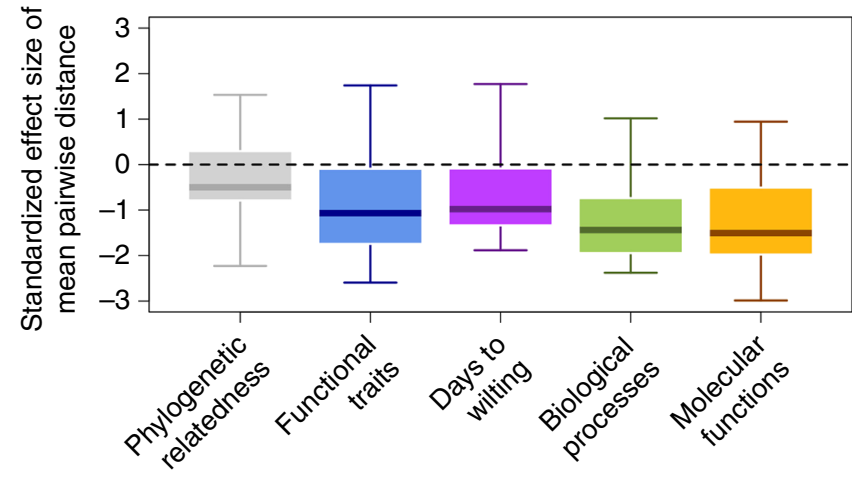

Fig. 1 Species similarity and tree co-occurrence in a Wisconsin natural forest plot. The results from null modeling analyses for $63020 \times 20 \mathrm{~m}$ subplots where a standardized effect size of the mean pairwise distance is on the $y$-axis and the type of similarity is on the $x$-axis. Negative standardized effect size values indicate species were more similar than expected given a random expectation and positive values indicate species were more dissimilar than expected. A linear mixed effects model ( $F=$ 425.19; $p<0.01$ ) with repeated measures and a Gaussian spatial covariance structure and subplot as a random effect followed by a Tukey Test showed that the boxplots were all significantly different from one another $(p<0.001)$

whether similar species, based on wilting, functional traits or relatedness, non-randomly co-occurred. These results were then compared to the results from the analogous gene expression analyses.

We hypothesized that if drought is a key driver of species distributions, performance, and ultimately local co-occurrence, then species with similar overall gene set enrichment in response to experimental drought should naturally co-occur. Second, we hypothesized that those GO molecular functions and GO biological processes related to drought and desiccation will be strong predictors of natural species co-occurrence. Third, we hypothesized that transcriptomic similarity in response to drought would be most strongly linked with natural cooccurrence.

Species with similar patterns of gene set enrichment in response to experimental drought across GO molecular functions and GO biological processes non-randomly co-occurred in nature (Fig. 1). This is indicated by the low standardized effect size (S.E. S.) values from the null model analyses. The result was robust to choice of the spatial scale of analysis, how species were clustered based upon the results from gene set enrichment analyses (Methods). Furthermore, we found little-to-no detectable phylogenetic signal in gene set enrichment (Tables 1, 2). In sum, similarity in the overall transcriptomic response between tree species to experimental drought is strongly linked to their cooccurrence in a natural stand. This indicates that differential transcriptomic responses to drought may be leading to differential distributions across hydraulic environments. The Wisconsin plot occurs on complex glacial outwash topography with wide variation in local substrate conditions, ranging from well-drained sandy loams on ridgetops and slopes to poorly-drained muck in depressions and drainage ways ${ }^{20}$. To investigate the local influence of response to drought in more detail, we mapped the distribution of S.E.S. values in the forest at the scale of $20 \times 20 \mathrm{~m}$ for both GO biological processes and GO molecular functions. We correlated these maps with maps of volumetric soil water content using a Pearson's correlation with a $p$-value estimated by a torus translation randomization ${ }^{21}$ that maintained the observed spatial auto correlation in the data. Soil water content was negatively correlated with both the GO biological process $(r=$
0.627; $p<0.005)$ and GO molecular function $(r=0.712 ; p<$ $0.005)$ S.E.S. maps (Table 3). In other words, more xeric soils in the forest plot are where co-occurring species are the most nonrandomly similar in their gene set enrichment in response to experimental drought (Fig. 1). From this we infer that species with similar responses to drought may be partitioning soil hydraulic gradients in this forest.

Gene ontology terms associated with species co-occurrence. The exact GO biological processes and GO molecular functions that were most closely tied to patterns of species co-occurrence were considered next. The top GO biological process ontologies where gene set enrichment similarity was most strongly linked to tree co-occurrence were response to water deprivation (GO:0009414). The next two most important were response to jasmonic acid (GO:0009753) and response to oxidative stress (GO:0006979) (Table 1). While we caution against overinterpretation of these results given that the GO annotations were generated in model organisms ${ }^{17}$, we do note that these gene ontologies have established linkages with water stress ${ }^{22-27}$. For example, during water stress the closure of stomata and excess light cause the photosynthetic apparatus to become over-reduced leading to photoinhibition and oxidative stress ${ }^{22}$. Additionally, the interplay between jasmonic acid and ABA signaling has been functionally demonstrated in Arabidopsis species under drought $^{28,29}$. Response to abscisic acid (GO:0009767) was the 11 th ranked biological process GO in our results (Table 1), where species that had similar gene set enrichment for this GO tended to co-occur.

The top GO molecular functions where similar gene set enrichment was linked to species co-occurrence were chitinase activity (GO:0004568), copper ion binding (GO:0005507), and transporter activity (GO:0005215) (Table 2). As with the biological processes, the most important molecular functions linked to tree co-occurrence are linked with plant stress ${ }^{30-32}$. In sum, we were able to quantify the transcriptomic response of species to experimental drought and using this information we successfully identified the biological process and molecular function GO terms that were most strongly linked with natural tree co-occurrences.

Transcriptomes outperform other predictors of co-occurrence. The above results suggest that water availability is a key driver of local tree species distributions and patterns of co-occurrence in this Wisconsin forest. Quantifying the dynamic response of plants to key environmental drivers provides important information not captured in more traditional functional trait-based studies of plant communities that have often focused on a few traits and not plastic responses. We compared the S.E.S. values from the gene set enrichment analyses to analogous S.E.S. values calculated using days to wilting data, functional trait similarity and phylogenetic relatedness. The results show that gene set enrichment $\mathrm{S}$. E.S. values were significantly lower than those calculated from wilting, trait, or relatedness data (Fig. 1). Next, we correlated the wilting, trait, and phylogenetic S.E.S. values with soil water content as done with the expression data. While these S.E.S. values were significantly correlated with soil water content, the relationships were not as strong as those for the S.E.S. values from the expression data (Table 3; Supplementary Table 4). This demonstrates that our transcriptomic analyses of gene expression were better at detecting non-random spatial co-occurrence in a natural stand of trees than were analyses of functional traits and phylogenetic relatedness. Transcriptomic analyses were further able to identify the key biological processes and molecular functions underlying this co-occurrence. 


\section{Table 1 The biological process gene ontologies analyzed in this study}

\begin{tabular}{|c|c|c|c|c|}
\hline $\begin{array}{l}\text { Gene ontology-biological } \\
\text { process }\end{array}$ & Description & $\begin{array}{l}\text { Mean standardized effect } \\
\text { size of the mean pairwise distance }\end{array}$ & D & $p$-value \\
\hline GO:0009414 & Response to water deprivation & -1.36 & 1.28 & 0.771 \\
\hline GO:0009753 & Response to jasmonic acid & -1.21 & 0.79 & 0.28 \\
\hline GO:0010200 & Response to chitin & -1.17 & 1.10 & 0.574 \\
\hline GO:0009611 & Response to wounding & -1.04 & 0.90 & 0.355 \\
\hline GO:0006629 & Lipid metabolic process & -0.97 & 1.55 & 0.952 \\
\hline GO:0000186 & Activation of MAPKK activity & -0.91 & 1.29 & 0.758 \\
\hline GO:0006865 & Amino-acid transport & -0.90 & 1.28 & 0.769 \\
\hline GO:0009737 & Response to abscisic acid & -0.86 & 1.92 & 0.841 \\
\hline GO:0010363 & $\begin{array}{l}\text { Regulation of plant-type hypersensitive } \\
\text { response }\end{array}$ & -0.78 & 1.49 & 0.941 \\
\hline GO:0006869 & Lipid transport & -0.78 & 0.52 & 0.128 \\
\hline GO:0010075 & Regulation of meristem growth & -0.76 & 0.75 & 0.268 \\
\hline GO:0006098 & Pentose-phosphate shunt & -0.67 & 1.82 & 0.977 \\
\hline GO:0009744 & Response to sucrose & -0.63 & 1.16 & 0.498 \\
\hline GO:0009860 & Pollen tube growth & -0.62 & 1.48 & 0.72 \\
\hline GO:0015996 & Chlorophyll catabolic process & -0.61 & 0.15 & 0.173 \\
\hline GO:0006633 & Fatty acid biosynthetic process & -0.61 & 0.13 & 0.164 \\
\hline GO:0006598 & Polyamine catabolic process & -0.60 & -1.69 & 0.011 \\
\hline GO:0010025 & Wax biosynthetic process & -0.60 & 2.16 & 0.876 \\
\hline GO:0006457 & Protein folding & -0.58 & 1.82 & 0.805 \\
\hline GO:0009269 & Response to desiccation & -0.55 & 1.73 & 0.804 \\
\hline GO:0009624 & Response to nematode & -0.52 & 0.48 & 0.124 \\
\hline GO:0009987 & Cellular process & -0.42 & 3.55 & 0.551 \\
\hline GO:0009615 & Response to virus & -0.42 & 6.22 & 0.819 \\
\hline GO:0000226 & Microtubule cytoskeleton organization & -0.41 & 4.46 & 0.795 \\
\hline GO:0016556 & mRNA modification & -0.37 & 6.56 & 0.914 \\
\hline GO:0006470 & Protein dephosphorylation & -0.37 & 1.33 & 0.437 \\
\hline GO:0006412 & Translation & -0.21 & 1.10 & 0.575 \\
\hline GO:0006857 & Oligopeptide transport & -0.09 & 1.45 & 0.891 \\
\hline GO:0009409 & Response to cold & 0.00 & 1.60 & 0.971 \\
\hline GO:0006073 & Cellular glucan metabolic process & 0.00 & 1.22 & 0.71 \\
\hline GO:0008152 & Metabolic process & 0.33 & 1.51 & 0.705 \\
\hline GO:0016310 & Phosphorylation & 0.37 & 0.86 & 0.326 \\
\hline GO:0006950 & Response to stress & 0.42 & -0.10 & 0.006 \\
\hline GO:0008272 & Sulfate transport & 0.43 & 0.94 & 0.385 \\
\hline GO:0009607 & Response to biotic stimulus & 0.49 & 0.73 & 0.253 \\
\hline GO:0009664 & Plant-type cell wall organization & 0.52 & 1.34 & 0.831 \\
\hline GO:0006351 & Transcription, DNA-templated & 0.73 & -1.13 & 0.278 \\
\hline GO:0019252 & Starch biosynthetic process & 0.77 & -1.37 & 0.278 \\
\hline GO:0005975 & Carbohydrate metabolic process & 0.83 & 0.06 & 0.015 \\
\hline GO:0006468 & Protein phosphorylation & 0.96 & -0.44 & $<0.001$ \\
\hline GO:0006952 & Defense response & 1.02 & 1.22 & 0.724 \\
\hline GO:0006355 & Regulation of transcription, DNA-templated & 1.04 & 1.05 & 0.508 \\
\hline GO:0006200 & ATP catabolic process & 1.44 & 1.04 & 0.462 \\
\hline GO:0015706 & Nitrate transport & 3.17 & -5.04 & 0.265 \\
\hline
\end{tabular}

The first two columns provide the gene ontology identifier and description. The third column provides the average of the standardized effect size of the mean pairwise distance values for $63020 \times 20 \mathrm{~m}$ subplots. Negative standardized effect size values indicate species with more similar than expected gene set enrichment in response to drought are naturally co-occurring. The fourth column presents the $D$ statistic, where a value of 1 indicates phylogenetic signal in the expression data (i.e., consistent with Brownian motion evolution on the phylogeny) and values higher than 1 indicate increasingly labile evolution. The final column presents the $p$-value for the $D$ statistic 
Table 2 The molecular function gene ontologies analyzed in this study

\begin{tabular}{|c|c|c|c|c|}
\hline $\begin{array}{l}\text { Gene ontology-molecular } \\
\text { function }\end{array}$ & Description & $\begin{array}{l}\text { Mean standardized effect size of the mean } \\
\text { pairwise distance }\end{array}$ & D & $p$-value \\
\hline GO:0004568 & Chitinase activity & -1.34 & 0.29 & 0.031 \\
\hline GO:0005507 & Copper ion binding & -1.32 & 0.04 & 0.011 \\
\hline GO:0005215 & Transporter activity & -1.11 & 1.23 & 0.698 \\
\hline GO:0003677 & DNA binding & -1.10 & 1.28 & 0.739 \\
\hline GO:0004553 & $\begin{array}{l}\text { Hydrolase activity, hydrolyzing O-glycosyl } \\
\text { compounds }\end{array}$ & -1.10 & -0.56 & $<0.001$ \\
\hline GO:0003700 & $\begin{array}{l}\text { Transcription factor activity, sequence-specific } \\
\text { DNA binding }\end{array}$ & -1.07 & 0.88 & 0.343 \\
\hline GO:0008171 & O-methyltransferase activity & -0.79 & 0.83 & 0.284 \\
\hline GO:0004190 & Aspartic-type endopeptidase activity & -0.69 & 1.88 & 0.975 \\
\hline GO:0009055 & Electron carrier activity & -0.69 & 1.50 & 0.881 \\
\hline GO:0005509 & Calcium ion binding & -0.68 & 1.20 & 0.665 \\
\hline GO:0016491 & Oxidoreductase activity & -0.67 & 2.04 & 0.992 \\
\hline GO:0003825 & $\begin{array}{l}\text { Alpha,alpha-trehalose-phosphate synthase (UDP- } \\
\text { forming) activity }\end{array}$ & -0.66 & 1.58 & 0.907 \\
\hline GO:0016301 & Kinase activity & -0.62 & 2.08 & 0.907 \\
\hline GO:0000166 & Nucleotide binding & -0.60 & 1.35 & 0.615 \\
\hline GO:0015416 & $\begin{array}{l}\text { Organic phosphonate transmembrane-transporting } \\
\text { ATPase activity }\end{array}$ & -0.60 & 2.04 & 0.891 \\
\hline GO:0008271 & $\begin{array}{l}\text { Secondary active sulfate transmembrane } \\
\text { transporter activity }\end{array}$ & -0.59 & 0.91 & 0.382 \\
\hline GO:0008810 & Cellulase activity & -0.58 & 0.16 & 0.142 \\
\hline GO:0004185 & Serine-type carboxypeptidase activity & -0.57 & 0.73 & 0.302 \\
\hline GO:0005516 & Calmodulin binding & -0.56 & 1.67 & 0.799 \\
\hline GO:0008289 & Lipid binding & -0.56 & -0.71 & 0.037 \\
\hline GO:0004871 & Signal transducer activity & -0.42 & 5.18 & 0.798 \\
\hline GO:0016168 & Chlorophyll binding & -0.42 & 4.56 & 0.82 \\
\hline GO:0005351 & Sugar:proton symporter activity & -0.42 & 6.21 & 0.952 \\
\hline GO:0016758 & Transferase activity, transferring hexosyl groups & -0.42 & 2.62 & 0.502 \\
\hline GO:0004650 & Polygalacturonase activity & -0.41 & -6.26 & 0.133 \\
\hline GO:0004872 & Receptor activity & -0.41 & 4.80 & 0.722 \\
\hline GO:0008168 & Methyltransferase activity & -0.41 & -7.22 & $<0.001$ \\
\hline GO:0009922 & Fatty acid elongase activity & -0.41 & 4.63 & 0.807 \\
\hline GO:0003723 & RNA binding & -0.41 & -3.57 & 0.13 \\
\hline GO:0008422 & Beta-glucosidase activity & -0.41 & -1.43 & 0.334 \\
\hline GO:0004806 & Triglyceride lipase activity & -0.40 & 4.72 & 0.607 \\
\hline GO:0005488 & Binding & -0.40 & 3.34 & 0.537 \\
\hline GO:0005315 & $\begin{array}{l}\text { Inorganic phosphate transmembrane transporter } \\
\text { activity }\end{array}$ & -0.39 & 3.69 & 0.496 \\
\hline GO:0015297 & Antiporter activity & -0.39 & 6.94 & 0.92 \\
\hline GO:0003993 & Acid phosphatase activity & -0.36 & 1.20 & 0.424 \\
\hline GO:0005524 & ATP binding & 0.49 & 1.54 & 0.95 \\
\hline GO:0004842 & Ubiquitin-protein transferase activity & 0.66 & -1.83 & 0.29 \\
\hline GO:0004672 & Protein kinase activity & 0.68 & 0.50 & 0.115 \\
\hline GO:0015198 & Oligopeptide transporter activity & 0.77 & -1.33 & 0.28 \\
\hline GO:0003899 & DNA-directed RNA polymerase activity & 0.84 & -1.67 & 0.275 \\
\hline GO:0003735 & Structural constituent of ribosome & 0.90 & 1.08 & 0.551 \\
\hline GO:0004674 & Protein serine/threonine kinase activity & 0.97 & 0.54 & 0.115 \\
\hline GO:0004091 & Carboxylic ester hydrolase activity & 1.13 & 0.96 & 0.435 \\
\hline GO:0003824 & Catalytic activity & 1.43 & -0.30 & 0.009 \\
\hline GO:0004180 & Carboxypeptidase activity & 3.34 & -2.55 & 0.255 \\
\hline
\end{tabular}

The first two columns provide the gene ontology identifier and description. The third column provides the average of the standardized effect size of the mean pairwise distance values for $63020 \times 20 \mathrm{~m}$ subplots. Negative standardized effect size values indicate species with more similar than expected gene set enrichment in response to drought are naturally co-occurring. The fourth column presents the $D$ statistic where a value of 1 indicates phylogenetic signal in the expression data (i.e., consistent with Brownian motion evolution on the phylogeny) and values higher than 1 indicate increasingly labile evolution. The final column presents the $p$-value for the $D$ statistic

Lastly, we considered the possibility that the species in our system spatially co-occurred due to their tolerances to other abiotic stressors that were not considered. For example, shade tolerance and water-logging tolerance are two additional important drivers of tree distributions ${ }^{33}$. Thus, we used a compilation of temperate tree shade, water-logging, and drought tolerance data from Niinemets and Valladares ${ }^{33}$ to address this possibility. Specifically, for each species we obtained their tolerances and calculated a similarity between species for each tolerance and conducted null modeling and soil water content correlative analyses just like we did for the expression, wilting, trait, and phylogenetic data (Methods). The results show that species with dissimilar shade and water-logging tolerances tend to co-occur and those with similar drought tolerances tend to cooccur (Fig. 2). Thus, tolerances to other factors are also significant drivers of tree distributions and co-occurrence in this forest. However, the effect sizes (S.E.S. values) are larger for drought tolerance, indicating that drought is a stronger predictor of tree co-occurrence in our study plot than the two other tolerances using an independent data source. We also note that the effect 
Table 3 The relationship between community dispersion and soil water content in the forest dynamics plot using point soil core data

\section{Type of dispersion}

Phylogenetic relatedness

$r$

Functional traits

$0.514^{\star}$

Days to wilting

$0.424^{*}$

GO biological processes

$0.612^{\star}$

0.627

GO molecular functions $\quad 0.712^{\star}$

Shade tolerance $\quad 0.193^{\star}$

Water-logging tolerance $\quad 0.019$

Drought tolerance $\quad 0.402^{\star}$

The Pearson's correlation between the S.E.S. of the MPD values for $20 \times 20 \mathrm{~m}$ subplots for those subplots where soil cores were taken. Significance, $p<0.05$ indicated with an asterisk, was assessed by generating a null distribution of expected Pearson's $r$ values using a torus translation

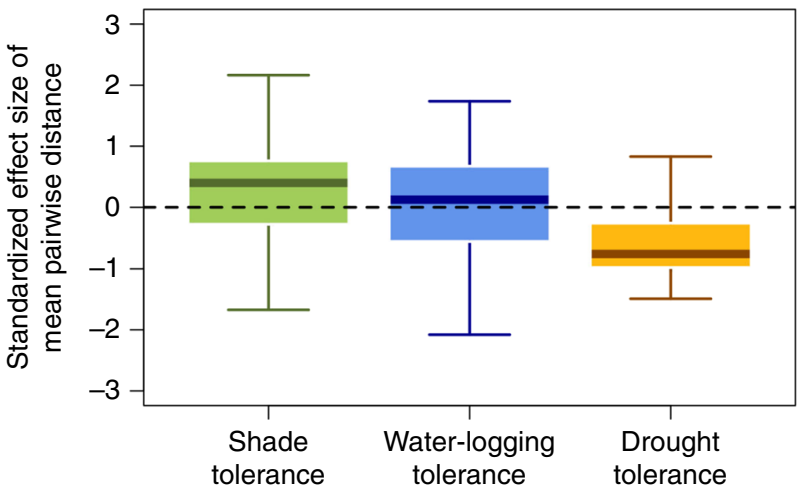

Fig. 2 Environmental tolerance similarity and tree co-occurrence in a natural forest plot. The results from the analyses for $63020 \times 20 \mathrm{~m}$ subplots, where a standardized effect size of the mean pairwise distance is on the $y$-axis and the type of similarity is on the $x$-axis. Negative standardized effect size values indicate species were more similar in their tolerances than expected given a random expectation and positive values indicate species were more dissimilar than expected

sizes from the expression data (Fig. 1) are larger than those generated from the drought tolerance data ${ }^{33}$ (Fig. 2), indicating that the expression data capture additional important information. Furthermore, while shade tolerance and drought tolerance $S$. E.S. values were correlated with soil water content, the strength of the correlations was weaker than that for expression S.E.S. values (Table 3; Supplementary Table 4).

\section{Discussion}

Tree species distributions and co-occurrences are the result of the interaction between organismal function and environmental drivers, both of which are temporally dynamic. Here we have focused on the dynamic response of species to drought and its relationship to natural tree co-occurrence. Our focus on drought stems from previous research suggesting that drought governs species distributions across spatial scales ${ }^{2,3}$ and ecosystem flux ${ }^{4,5}$, and the impact of drought on forested ecosystems is expected to increase in frequency and intensity in the future ${ }^{6}$. We have presented a community transcriptomics-based approach that provides a broad assay of plant function and its dynamic response to changing conditions. The results show that species with similar transcriptomic responses to experimental drought tend to nonrandomly co-occur along the soil water gradient in a natural forest stand. We were further able to identify the individual biological process and molecular function ontological domains most closely associated with natural co-occurrence. This analysis suggests that species with similar transcriptomic responses to decreasing water availability may have superior demographic performance, but future analyses that will leverage continued monitoring of this forest will be needed to test this directly. Our results also show that transcriptomic similarity was the strongest indicator of natural species co-occurrence compared to functional trait similarity and phylogenetic relatedness. Although measures of functional traits and phylogenetic similarity may provide useful initial insights into the drivers of community structure, a significant degree of important information is not captured using these measures. We do caution that the use of GO terms from distantly related model species is not a panacea and has weaknesses. As the technical, computational, and financial barriers to the use of transcriptomic approaches are lowered, particularly for non-model systems, trait-based community ecology will be poised to dramatically expand its capacity to broadly and dynamically assay functional diversity ${ }^{34-36}$. We expect that this will provide exciting new mechanistic insights into how phenotypes interact with their environments to drive community structure and dynamics now and into the future.

\section{Methods}

Forest dynamics plot and biodiversity data. This study aimed to quantify the linkages between gene expression similarity in response to experimental drought and the spatial co-occurrence of tree species in a natural community. To this end we utilized data from the Wabikon Lake Forest Dynamics Plot-a Smithsonian ForestGeo Network site ${ }^{37}$. The Wabikon Lake plot is 25.2 hectares in area and is located in the Chequamegon-Nicolet National Forest in Wisconsin, USA. The forest plot is censused every 5 years, where all free-standing woody individuals $\geq 1$ $\mathrm{cm}$ in diameter at $130 \mathrm{~cm}$ from the ground are identified, spatially mapped and have their diameters measured. The study used data from the $63020 \times 20 \mathrm{~m}$ subplots in the forest plot.

A soil survey of the plot was conducted in the summer of 2015, where soil volumetric moisture content was quantified by weighing fresh and dried soil cores Soil cores were taken from the upper $10 \mathrm{~cm}$ of mineral soil using a regular $40 \times 40$ $\mathrm{m}$ grid inside the plot. Additionally, at every other $40 \times 40 \mathrm{~m}$ point soil samples were taken at 2,8 , and $20 \mathrm{~m}$ from that point in a randomly chosen direction (Supplementary Fig. 3). This soil sampling design follows that of other forest dynamics plot in our research network ${ }^{16}$. Samples were taken over a 4 -day period with no periods of rainfall during or immediately preceding the sampling period. In total, 252 samples were collected. A map of soil moisture content was generated by constructing spatial semivariograms and kriging at the resolution of $20 \times 20 \mathrm{~m}$ such that a soil moisture value was estimated in each subplot.

This study used the first census of the plot performed in 2008 and focused on the 21 gymnosperm and angiosperm species that dominate the canopy

(Supplementary Table 1). The only species that reaches a diameter $>10 \mathrm{~cm}$ and has more than 100 individuals in the whole plot that was not considered in this study was Fraxinus nigra (Oleaceae). This species could not be sourced and grown in the greenhouse due to concerns regarding the spread of the Emerald Ash Borer (Agrilus planipnnis), which is currently decimating Fraxinus populations in the Upper Midwestern USA.

Our previous work in this forest has generated a molecular phylogeny and functional trait data for the species. Specifically, a three-locus barcode phylogenetic chronogram containing the species from this plot and 14 other ForestGeo plots was presented in Erickson et al. ${ }^{19}$ For this study, the Erickson et al. phylogeny was pruned to contain only the 21 study species. The functional trait data for this study comes from Swenson et al. ${ }^{18}$ in which foliar N, foliar P, specific leaf area, wood density, seed mass, leaf area, and maximum height were quantified for each species. Leaf nutrient and specific leaf area data were used to represent the welldocumented leaf economics spectrum ${ }^{38}$, where species are arrayed along a spectrum of low investment and long leaf lifespans vs. high investment and short leaf lifespans. Wood density was used to represent the wood economics spectrum $^{39}$, where species have low construction costs and mortality rates vs. high construction costs and mortality rates. Seed mass was used to represent a fundamental tradeoff between producing many poorly-provisioned offspring vs. a few well-provisioned offspring ${ }^{40}$. Leaf area was used to represent the organ level area for resource capture, which correlates with leaf temperatures ${ }^{41}$ and maximum height was used to estimate the adult light niche of the species ${ }^{42}$. In this study, as in our other work ${ }^{18}$, the trait data were used in a principle component (PC) analysis (Supplementary Table 2) to account for co-variation and the first two PC axes were used in the subsequent analyses of trait dispersion. 
Drought experiment. The drought experiment utilized 1-year old seedlings for all species. Seeds were originally sourced from the Upper Peninsula of Michigan and northern Wisconsin by Alpha Nurseries, Inc., Holland, Michigan within $200 \mathrm{~km}$ of the forest dynamics plot. While sampling from within the plot or immediately adjacent to the plot would have been ideal, it was not possible in the present student and likely introduces some bias. However, the geographic and climatic distance of collection sites from the forest plot is generally small indicating that local adaptation to dramatically different hydraulic environments was not likely. The relatedness of conspecific individuals is not known thereby limiting our ability to consider this degree of variation across species. However, multiple seeds were typically collected from a single adult individual indicating that there is likely a higher probability that the individuals studied are half-siblings. The seedlings were grown for one growing season prior to transplantation into the greenhouses at Michigan State University in the spring of 2014. After leaf emergence, the plants were grown for 8 weeks under well-watered conditions in the same room. Next, 10 plants of each species were selected for the drought treatment and the remaining plants continued under the same watering regime and were used as controls. The plants in the drought treatment no longer received water.

A LiCor-6400 was used to quantify leaf stomatal conductance and photosynthetic rate on control and droughted plants. Species-level distributions of stomatal conductance and photosynthetic rates were generated from control plants that could be compared to individual plants in the drought treatment. For control plants, immediately following LiCor measurements the same leaf was flash frozen in liquid nitrogen and transferred to a $-80^{\circ} \mathrm{C}$ freezer. Plants in the drought treatment were monitored daily for signs of wilt. If the plant appeared wilted, then its stomatal conductance and photosynthetic rate were quantified. If the values were $>2$ standard deviations from the mean of the control distribution for that species, leaves were flash frozen and placed in the freezer. The number of days to wilt per individual per species was recorded and an average days to wilting, and therefore days to tissue sampling, was recorded. For gymnosperms, where there was no visual wilting, a random individual was selected every 1 day for photosynthesis measurements. If that individual was significantly droughted, the other individuals from that species in the drought experiment were also measured and flash froze. Thus, the days to wilting for these species is the number of days from the start of the experiment to sampling and we refer to this as days to wilting in the paper for simplicity. The average difference in the days to wilting between species was $<20$ days. Thus, there were no major ontogenetic changes between individuals of species wilting early in the study and those wilting later in the study and therefore biases due to ontogeny were not a concern, whereas they may have been a concern if our study organisms had shorter life cycles.

RNA sequencing and expression analyses. A reference transcriptome to which we could map expression data was generated for each species. The reference for each species was generated using four control individuals and three droughted individuals. For all samples, RNA was extracted using an RNeasy Plant Mini Kit (Qiagen; Valencia, California) with RNA quality and quantity measured using a NanoDrop 2000 spectrophotometer and an Agilent Bioanalyzer 2100. RNAseq was performed at the Beijing Genomics Institute, Shenzhen, China using Illumina 2000 HiSeq platform using $100 \mathrm{bp}$ paired-end reads and with eight samples per lane. Thus, there were ultimately seven samples used per species ( 4 control and 3 drought) and all samples were sequenced on the sample platform and at the same depth. The average number of clean reads per sample across all species was 60 million with a range of 51-68 million reads (Supplementary Table 1).

Reference transcriptomes were assembled de novo using Trinity ${ }^{43}$. The N50 values for the species assemblies ranged from 1291 to 1835 (Supplementary Table 1). References were annotated against the NCBI nr and SwissProt databases using blastx where only the top hit was retained if multiple significant hits $(E-$ values $<1 \times 10^{-5}$ ) were detected and Blast2 $\mathrm{GO}^{44}$ was utilized to assign GO terms for biological processes and molecular functions. SOAPaligner ${ }^{45}$ was used to map reads from the four control and three drought samples per species to their respective reference transcriptomes with no more than five mismatched bases allowed for mapping. The counts data were then used in edge ${ }^{46}$ to quantify reads per kilobase of transcript per million mapped reads (RPKM) for the two groups (control and drought) using all samples from the group. We then used edgeR to quantify differential expression ( $\log _{2}$ fold changes of \pm 1 were used as cutoffs) and adjusted $p$-values using a Benjamini-Hochberg false discovery rate.

Next, genes were grouped by GO terms that were found across all 21 species in the experiment. In other words, if a GO term was found in less than all 21 species it was not included in any downstream analyses. Thus, there was GO information for a particular species not found in all other species and therefore could not be readily compare. For example, Acer saccharum had more than 1000 GO terms identified for biological processes and molecular functions, but fewer than 100 of these could be compared across all species (Tables 1,2). Future work taking a functional phylogenomic approach that can construct individual gene trees or that looks at GO terms where only some species are represented should be conducted and may prove informative. However, that is beyond the scope of the present study and the following statistical tests and the false discovery rate adjustments were based upon only those GO terms found in all 21 species. A Fisher's exact test was then used to assess gene set enrichment for each GO term again using a Benjamini-Yekutieli false discovery rate adjustment ${ }^{47}$. The odds ratio was then clustered across species using hierarchical clustering (Supplementary Figs. 1, 2). Similarly, species were hierarchically clustered based upon whether they were significantly enriched or not (Supplementary Figs. 1, 2). The branch lengths from these hierarchical clustering dendograms using the significance data were used for downstream analyses of similarity and natural co-occurrence where species with less branch length separating them had more similar overall gene set enrichment in response to experimental drought than did species with more branch length separating them. Subsequent analyses also investigated similarity using individual GO terms where similarity between species was based upon whether they had the same (significant-significant or non-significant-non-significant) or different (significant-non-significant or non-significant-significant) gene set enrichment in response to experimental drought.

Community dispersion analyses. We quantified whether tree species in the Wabikon Lake forest plot non-randomly co-occurred with respect to phylogenetic relatedness, functional trait similarity, environmental tolerance similarity ${ }^{33}$, days to wilting similarity, and similarity in gene set enrichment in response to experimental drought using biological process and molecular function GO categories The environmental tolerance data were taken from Niinemets and Valladares ${ }^{33}$ who estimated the relative tolerance of temperate tree species, inclusive of our study species, to drought, shade, and water-logging. The rationale for using this data was that it provided an alternative and independent assay of drought tolerance and allowed us to investigate if other axes of environmental tolerance (i.e., shade and water-logging) were as or more important in our study system. The days to wilting data were used independently of the functional trait data as a separate and experimental assay of drought tolerance. All analyses used a mean pairwise distance (MPD) between all individual trees in a sample as:

$$
\operatorname{MPD}=\frac{\sum_{i}^{n} \sum_{j}^{n} \delta_{i, j} f_{i} f_{j}}{\sum_{i}^{n} \sum_{j}^{n} f_{i} f_{j}}
$$

where $f_{i}$ is the number of individuals of species $i, f_{j}$ is the number of individuals of species $j$, and $\delta_{i, j}$ is the phylogenetic distance between two taxa, the Euclidean distance between two taxa positioned on the two PC axes derived from the functional trait, tolerance or days to wilting data, the distance between two species on the hierarchical clustering of gene set enrichment for all GO molecular functions (Supplementary Fig. 1) or the distance between two species on the hierarchical clustering of gene set enrichment for all GO biological processes (Supplementary Fig. 2). These observed values were then compared to a null distribution of MPD values generated by randomizing the names of taxa on the respective data sets 999 times and a S.E.S. was calculated as:

$$
\text { S.E.S. }=\frac{\text { MPD }_{\text {obs }}-\text { meanMPD }_{\text {null }}}{\text { st.devMPD }} \text { null }
$$

where $M P D_{\text {obs }}$ is the observed MPD value, meanMPD $D_{\text {null }}$ is the mean MPD value in the null distribution, and st.devMPD null is the standard deviation of the null distribution ${ }^{48}$. A negative S.E.S. value indicated co-occurring species were more similar than expected and a positive value indicated co-occurring species were less similar than expected. The analyses were conducted using $20 \times 20 \mathrm{~m}$ subplots, but similar results at the $40 \times 40 \mathrm{~m}$ and $100 \times 100 \mathrm{~m}$ scales were found. We show only the $20 \times 20 \mathrm{~m}$ results because they were consistent with other scales and because our elevation data set was collected at this scale making our downstream spatial analyses at this scale possible.

Next, we performed the above analyses using a single GO biological process or molecular function category at a time (i.e., a single column in Supplementary Figs. 1 and 2). In these analyses the $\delta_{i, j}$ value in the MPD calculation was 0 if the two species both had significant or non-significant gene set enrichment for that category and 1 if one species had a significant enrichment while the other did not. As with the above analyses, a null model was implemented and a S.E.S. value was calculated. We then ranked GO categories based on their average S.E.S. value across all $20 \times 20 \mathrm{~m}$ subplots to determine which individual biological processes and molecular functions were those where similarity in gene set enrichment was most strongly related to tree co-occurrence.

Spatial analyses. To test the expectation that similarity in gene expression response to experimental drought would be most evident in natural species assemblages on more xeric edaphic environments, we utilized the soil water content data from the 252 soil cores taken in the plot. Specifically, we correlated the S E.S. values for the phylogenetic, functional trait, days to wilting, tolerances, $\mathrm{GO}$ biological processes, and the GO molecular functions from those $20 \times 20 \mathrm{~m}$ subplots that had soil cores taken with the soil water content data and used a torus translation that accounts for spatial autocorrelation to quantify a $p$-value ${ }^{21}$. In a secondary analysis, we estimated the soil water content in each of the $63020 \times 20 \mathrm{~m}$ subplots using kriging. The kriged values were then correlated with the S.E.S. values and $p$-values were again assessed using a torus translation.

Phylogenetic signal analyses. A concern regarding our approach and arising from our results could be that a strong phylogenetic signal in the data may be biasing our results and our interpretations. For example, if gymnosperms tend to 
cluster together in their gene expression responses, in general or for specific GO terms, and tend to co-occur in the forest plot for reasons not linked to drought response, we may have flawed inferences regarding the importance of gene expression response to drought. Thus, if there is phylogenetic signal in the data, a phylogenetic comparative method should be used. We quantified the degree of phylogenetic signal in the expression data in two different ways. First, to quantify the phylogenetic signal in the overall similarity in gene set enrichment we utilized a Mantel test relating phylogenetic distance to the distance between two species on the gene set enrichment hierarchical clustering dendrograms used for our dispersion analyses (Supplementary Figs. 1,2). If there was positive and significant relationship, then this would be evidence of phylogenetic signal.

Our second approach investigated individual GO terms. For this analysis, as in our dispersion analyses, species were coded as having a significant gene set enrichment for a specific GO term (1) or no significant enrichment (0). Thus, the coding of species was binary. Phylogenetic signal in binary data is best measured using the $D$ statistic of Fritz and Purvis ${ }^{49}$ where Brownian Motion trait evolution is where $D=1$, labile trait evolution is where $D>1$, and trait evolution more conservative than Brownian Motion is where $D<1$. The significant of the $D$-value is gauged using a permutation test, where observed values are randomized across the tips of the phylogeny 999 times and a null distribution of

$D$-values is estimated.

Data availability. Data are available in Dryad (https://doi.org/10.5061/dryad. q3j60) and NCBI-SRA (PRJNA413418). The authors declare that all other data supporting the findings of this study are available from the corresponding authors upon request.

Received: 16 May 2016 Accepted: 2 November 2017

Published online: 08 December 2017

\section{References}

1. McGill, B. J. et al. Rebuilding community ecology from functional traits. Trends Ecol. Evol. 21, 178-185 (2006).

2. Engelbrecht, B. M. J. et al. Drought sensitivity shapes species distribution patterns in tropical forests. Nature 447, 80-82 (2007).

3. Zang, C. et al. Patterns of drought tolerance in major European temperate forest trees: climatic drivers and levels of variability. Global Change Biol. 20, 3767-3779 (2014)

4. Phillips, O. L. et al. Drought-mortality relationships for tropical forests. New Phytol. 187, 631-646 (2010).

5. Doughty, C. E. et al. Drought impact on forest carbon dynamics and fluxes in Amazonia. Nature 519, 78-82 (2015).

6. Trenberth, K. E. et al. Global warming and changes in drought. Nat. Clim. Chang. 4, 17-22 (2014)

7. Kraft, N. J. B., Valencia, R. \& Ackerly, D. D. Functional traits and niche-based tree community assembly in an Amazonian forest. Science 322, 580-582 (2008).

8. Stahl, U., Reu, B. \& Wirth, C. Predicting species' range limits from functional traits for the tree flora of North America. Proc. Natl Acad. Sci. USA 111 13739-13744 (2014).

9. Swenson, N. G. The assembly of tropical tree communities-the advances and shortcomings of phylogenetic and functional trait analyses. Ecography 36, 264-276 (2013)

10. Diaz, S. et al. The global spectrum of plant form and function. Nature 529, 167-171 (2016)

11. Westoby, M. et al. Plant ecological strategies: some leading dimensions of variation between species. Annu. Rev. Ecol. Syst. 33, 125-159 (2002).

12. Webb, C. O., Cannon, C. H. \& Davies, S. J. in Tropical Forest Community Ecology (eds Carson, W. P. \& Schnitzer, S. A.) 79-97 (Wiley-Blackwell, West Sussex, 2008).

13. Cadotte, M. W., Albert, C. \& Walker, S. The ecology of differences: integrating evolutionary and functional distances. Ecol. Lett. 16, 1234-1244 (2013).

14. Paine, C. E. T. et al. Globally, functional traits are weak predictors of juvenile tree growth, and we do not know why. J. Ecol. 103, 978-989 (2015).

15. Kraft, N. J. B., Godoy, O. \& Levine, J. M. Plant functional traits and the multidimensional nature of species coexistence. Proc. Natl Acad. Sci. USA 112, 797-802 (2015)

16. Kunstler, G. et al. Plant functional traits have globally consistent effects on competition. Nature 529, 204-207 (2016).

17. Pavlidis, P., Jensen, J. D., Stephan, W. \& Stamatakis, A. A critical assessment of storytelling: gene ontology categories and the importance of validating genomic scans. Mol. Biol. Evol. 29, 3237-3248 (2012).

18. Swenson, N. G. et al. Phylogenetic and functional alpha and beta diversity in temperate and tropical tree communities. Ecology 93, 112-125 (2012).
19. Erickson, D. L. et al. Comparative evolutionary diversity and phylogenetic structure across multiple forest dynamics plots: a mega-phylogeny approach. Front. Genet. 5, e358 (2014).

20. Boelter, J. M., Elg, A. M. \& Barnes, J. R. Soil Survey of Forest County, Wisconsin (United States Department of Agriculture, Natural Resources Conservation Service and Forest Service, Washington, D.C., 2005).

21. John, R. et al. Soil nutrients influence spatial distributions of tropical tree species. Proc. Natl Acad. Sci. USA 104, 864-869 (2007).

22. Mittler, R. Oxidative stress, antioxidants and stress tolerance. Trends Plant Sci. 7, 405-410 (2002).

23. Bartoli, C. G. et al. Drought and watering-dependent oxidative stress: effect on antioxidant content in Triticum aestivum L. leaves. J. Exp. Bot. 50, 375-383 (1999).

24. Sharma, P. \& Dubey, R. S. Drought induces oxidative stress and enhances the activities of antioxidant enzymes in growing rice seedlings. Plant Growth Regul. 26, 209-221 (2005).

25. Creelman, R. A. \& Mullet, J. E. Jasmonic acid distribution and action in plants: regulation during development and response to biotic and abiotic stress. Proc Natl Acad. Sci. USA 92, 4114-4119 (1995).

26. Shinozaki, K. \& Yamaguchi-Shinozaki, K. Gene networks involved in drought stress response and tolerance. J. Exp. Bot. 58, 221-227 (2007).

27. Walia, H. et al. Large-scale expression profiling and physiological characterization of jasmonic acid-mediated adaptation of barley to salinity stress. Plant Cell Environ. 30, 410-421 (2007).

28. Yin, Y. et al. Involvement of OST1 protein kinase and PYR/PYL/RCAR receptors in methyl jasmonate-induced stomatal closure in Arabidopsis guard cells. Plant Cell Physiol. 57, 1779-1790 (2016).

29. Harb, A., Krishnan, A., Ambavaram, M. M. R. \& Pereira, A. Molecular and physiological analysis of drought stress in Arabidopsis reveals early responses leading to acclimation in plant growth. Plant Physiol. 154, 1254-1271 (2010).

30. Kasprzewska, A. N. Plant chitinases-regulation and function. Cell. Mol. Biol. Lett. 8, 809-824 (2003).

31. Yao, D. et al. Transcriptome analysis reveals salt-stress-regulated biological processes and key pathways in roots of cotton (Gossypium hirsutum L.). Genomics 98, 47-55 (2011).

32. Ranjan, A. et al. Comparative transcriptomic analysis of roots of contrasting Gossypium herbaceum genotypes revealing adaptation to drought. BMC Genomics 13, 1 (2012).

33. Niinemets, U. \& Valladares, F. Tolerance to shade, drought, and waterlogging of temperate Northern Hemisphere trees and shrubs. Ecol. Monogr. 74, 521-547 (2006)

34. Swenson, N. G. The functional ecology and diversity of tropical tree assemblages through space and time: from local to regional and from traits to transcriptomes. ISRN Forestry 2012, 743617 (2012).

35. Zambrano, J. et al. Neighborhood defense gene similarity effects on tree performance: a community transcriptomic approach. J. Ecol. 105, 616-626 (2017).

36. Swenson, N. G. \& Jones, F. A. Community transcriptomics, genomics and the problem of species co-occurrence. J. Ecol. 105, 563-568 (2017).

37. Davies, S. J. Smithsonian Tropical Research Institute-ForestGeo Network, http://www.forestgeo.si.edu (2017).

38. Wright, I. J. et al. The world-wide leaf economics spectrum. Nature 428, 821-827 (2004).

39. Chave, J. et al. Towards a worldwide wood economics spectrum. Ecol. Lett. 12, 351-366 (2009).

40. Venable, D. L. Packaging and provisioning in plant reproduction. Philos. Trans. R. Soc. Ser. B 351, 1319-1329 (1996).

41. Dolph, D. E. \& Dilcher, D. L. Variation in leaf size with respect to climate in Costa Rica. Biotropica 12, 91-99 (1980).

42. Kohyama, T. et al. Tree species differentiation in growth, recruitment and allometry in relation to maximum height in a Bornean mixed dipterocarp forest. J. Ecol. 91, 797-806 (2003).

43. Grabherr, M. G. et al. Full-length transcriptome assembly from RNA-seq data without a reference genome. Nat. Biotechnol. 29, 644-652 (2011).

44. Conesa, A. et al. Blast2GO: a universal tool for annotation, visualization and analysis in functional genomics research. Bioinformatics 21, 3674-3676 (2005).

45. Ruiqiang, L. et al. SOAP: short oligonucleotide alignment program. Bioinformatics 24, 713-714 (2008).

46. Robinson, M. D., McCarthy, D. J. \& Smyth, G. K. edgeR: a Bioconductor package for differential expression analysis of digital gene expression data. Bioinformatics 7, 139-140 (2010).

47. Benjamini, Y. \& Yekutieli, D. The control of the false discovery rate in multiple testing under dependency. Ann. Stat. 29, 1165-1188 (2001).

48. Swenson, N. G. Functional and Phylogenetic Ecology in R. Springer User Series (Springer, New York, 2014).

49. Fritz, S. A. \& Purvis, A. Selectivity in mammalian extinction risk and threat types: a new measure of phylogenetic signal in binary traits. Conserv. Biol. 24, 1042-1051 (2010). 


\section{Acknowledgements}

The work in this paper was funded by a United States National Science Foundation USAChina Dimensions of Biodiversity grant (DEB-1241136) awarded to N.G.S. and a Fellowship from the John Simon Guggenheim Memorial Foundation awarded to N.G.S. Y.I. was supported by JSPS Overseas Research Fellowships. Field research at the Wabikon Lake Plot was supported by funding from The 1923 Fund, the Smithsonian Institution's ForestGEO Program, and the Cofrin Center for Biodiversity at the University of Wisconsin-Green Bay. We thank D. Kramer and D.W. Schemske for sharing lab equipment and D.W. Schemske for providing greenhouse space.

\section{Author contributions}

N.G.S. and K.M. conceived the idea for this collaborative research project. N.G.S., Y.I., R. H., B.L.T., and A.W. coordinated data collection. N.G.S., M.N.U., and K.P. designed and implemented the analytical approach. N.G.S. wrote the paper with comments and edits provided by all co-authors.

\section{Additional information}

Supplementary Information accompanies this paper at https://doi.org/10.1038/s41467017-02034-w.

Competing interests: The authors declare no competing financial interests.
Reprints and permission information is available online at http://npg.nature.com/ reprintsandpermissions/

Publisher's note: Springer Nature remains neutral with regard to jurisdictional claims in published maps and institutional affiliations.

Open Access This article is licensed under a Creative Commons Attribution 4.0 International License, which permits use, sharing, adaptation, distribution and reproduction in any medium or format, as long as you give appropriate credit to the original author(s) and the source, provide a link to the Creative Commons license, and indicate if changes were made. The images or other third party material in this article are included in the article's Creative Commons license, unless indicated otherwise in a credit line to the material. If material is not included in the article's Creative Commons license and your intended use is not permitted by statutory regulation or exceeds the permitted use, you will need to obtain permission directly from the copyright holder. To view a copy of this license, visit http://creativecommons.org/ licenses/by/4.0/.

(C) The Author(s) 2017 